\title{
Artificial gauge induced dispersionless coupling for broadband photonic waveguide integration
}

\section{Wange Song}

Nanjing University

Ting Li

ShanghaiTech University

Shenglun Gao

Nanjing University

Zhizhang Wang

Nanjing University

Shengjie Wu

Nanjing University

\section{Chen Chen}

Nanjing University

\section{Shining Zhu}

National Laboratory of Solid State Microstructures and School of Physics, Nanjing University

https://orcid.org/0000-0002-3472-6497

\section{Yi Zou}

ShanghaiTech University

Tao Li ( $\nabla$ taoli@nju.edu.cn )

Nanjing University https://orcid.org/0000-0003-0049-471X

\section{Article}

Keywords: broadband photonic waveguide integration, photonic integration, coupling

Posted Date: June 1st, 2021

DOl: https://doi.org/10.21203/rs.3.rs-492856/v1

License: (1) This work is licensed under a Creative Commons Attribution 4.0 International License. Read Full License 


\title{
Artificial gauge induced dispersionless coupling for broadband photonic waveguide integration
}

\author{
Wange Song, ${ }^{1,2}$ Ting Li, ${ }^{3}$ Shenglun Gao, ${ }^{1,2}$ Zhizhang Wang,,${ }^{1,2}$ Shengjie Wu,${ }^{1,2}$ Chen Chen, ${ }^{1,2}$ Shining \\ Zhu, ${ }^{1,2}$ Yi Zou, ${ }^{3}$ and Tao $\mathrm{Li}^{1,2, *}$ \\ ${ }^{I}$ National Laboratory of Solid State Microstructures, Key Laboratory of Intelligent Optical Sensing and Manipulation, \\ Jiangsu Key Laboratory of Artificial Functional Materials, College of Engineering and Applied Sciences, Nanjing \\ University, Nanjing, 210093, China. \\ ${ }^{2}$ Collaborative Innovation Center of Advanced Microstructures, Nanjing, 210093, China. \\ ${ }^{3}$ School of Information Science and Technology, ShanghaiTech University, Shanghai, 201210, China. \\ *taoli@nju.edu.cn
}

Abstract: Coupling among waveguides plays an important role in photonic integration, while it usually suffers from large wavelength dispersion and structural sensitivity that brings difficulties in broadband and robust photonic chip devices. Here, we report a new strategy of dispersion engineering of coupled waveguides by artificial gauge field (AGF) with curved trajectories, which gives rise to a dispersionless broadband coupler function in high-density silicon waveguides (waveguide pitch $<\lambda / 2$ ). It is found that the artificial gauge field can generate an inverse dispersion to compensate for the dispersion of conventional waveguide coupling. As such, the coupling between the waveguides can be stable in a broad bandwidth. Based on this design, we demonstrate compact directional and $3 \mathrm{~dB}$ couplers that show broadband dispersionless coupling of light with wavelength from 1400 to $1650 \mathrm{~nm}$, which also exhibit robustness to considerably large structural variations $\sim 150 \mathrm{~nm}$ (75\% structural deviation). Furthermore, using the AGF coupler as the building block, we significantly demonstrate a three-level-cascaded waveguide network to route the broadband light to the desired ports, showing a tremendous advantage over the conventional counterparts. Our work exploits the artificial gauge field to integrated photonics and demonstrates the possibility of massive, broadband, robust and dense photonic integrations. 


\section{Introduction}

The ability to control light propagation in photonic integrated circuits is at the foundation of modern optical information processing and optoelectronic integrations, in which broadband, robust, low-loss, and densely integrated optical components are highly desirable for reliable and large-scale integration ${ }^{1-6}$. However, the optical diffraction limitation ${ }^{7}$ hinders the improvement of integrations, and the wavelength dispersion of optical component ${ }^{8}$ makes it difficult to achieve broadband property. Among various integrated photonic functions and devices, coupling devices are important building blocks and have been employed as optical routing, mode converters, and switches for high-speed communication and data processing ${ }^{9,10}$, which fundamentally rely on the evanescent wave coupling ${ }^{11}$. Usually, the devices based on evanescent waves coupling exhibit high structure-sensitive and narrowband performance especially within high-density integrations. Tremendous efforts have been made to control the evanescent wave coupling and realize the broadband property. e.g., the adiabatic structures ${ }^{12-14}$, inverse design methods ${ }^{15-18}$, metamaterials ${ }^{19-23}$, and topological designs ${ }^{24,25}$. However, the multiple desired qualities are difficult to reach at the same time, usually, one satisfied performance needs to sacrifice in other important aspects. For example, the adiabatic optical couplers exhibit broadband property but have a large footprint $(\sim 150 \mu \mathrm{m})^{14}$. The same is true for the topological inspired designs (footprint $\sim 80 \mu \mathrm{m})^{24}$. On the contrary, the inverse design methods usually have compact footprints, while the performances highly depend on the design optimization algorithm ${ }^{15-18}$. The metamaterial-assisted waveguides ${ }^{19-23}$, however, need very tiny structures that inevitably increase the insertion losses and make the fabrication tedious, which is insufficient and costly for many practical applications. As a result, there is a high demand for devices with new design principles involved.

By properly engineering a physical system, for example, through the geometric design of a system, artificial gauge field (AGF) can be generated to govern the effective dynamics of neutral particles (e.g. photons). As an important physical concept, artificial gauge field allows us to endow photonic systems with a wide range of intriguing phenomena and novel functions ${ }^{26-37}$. To be noted, the artificial gauge field created by curved waveguides exhibit high flexibility for manipulating the optical fields. For example, dynamic localizations $\mathrm{s}^{28-30}$, Floquet topological insulators ${ }^{31-33}$, and quantum simulations $\mathrm{s}^{34,35}$. Recently, light guiding has been realized by artificial gauge field in direct laser writing fused silica waveguides ${ }^{36,37}$. However, the system is a few centimeters and not suitable for compact integration. Besides, more functionalities such as 
light coupling and routing, are still need to be explored with the presence of gauge field, especially in highly dense integration. More recently, the subwavelength self-imaging in closely packed curved silicon waveguides by artificial gauge field has been demonstrated ${ }^{38}$, suggesting the possibility of utilizing artificial gauge field for the control of light at subwavelength scales and a promising application for integrated photonics.

Here, we develop a new dispersion controlling mechanism of near-field coupling by artificial gauge field, and experimentally demonstrate broadband and robust coupling in densely packed silicon waveguides. Due to the curve-introduced artificial gauge field, the wavelength dispersion of the systems exhibits a flat band, indicating a high insensitivity to the wavelength and structural variations. We designed and fabricated the artificial gauge field assisted coupler (AGF coupler) in silicon on insulator (SOI) integrated platform with compact footprint, which indeed exhibits broadband and robust performance against large structural fluctuations as compared with the conventional coupler. Furthermore, we experimentally demonstrate the broadband light coupling in a three-leveled cascaded AGF device network with an overwhelming advantage over the conventional devices in comparison, promising the potential in massive integrations. In addition, the AGF devices based on silicon waveguides introduce negligible insertion losses to photonic integrated circuits. Our integrated AGF waveguide devices could help to realize broadband, robust, massive and high-density photonic integration.

\section{Dispersionless coupling by artificial gauge field}

For simplicity, we start from a weakly coupled waveguides with sinusoidal trajectory, i.e., $x(z)=A \sin (2 \pi z / P+\varphi)$, as shown in Fig. 1a, which can introduce a well-defined artificial gauge field ${ }^{36}$. The equation of motion for a Bloch wave in this system writes

$$
i \partial_{z} \phi_{k}(z)=2 c \cos \left(\left(k_{x}+G(z)\right) d\right) \phi_{k}(z)
$$

where $G(z)=k_{0} \dot{x}(z)=\left(4 \pi^{2} A / P \lambda\right) \cos (2 \pi z / P+\varphi)$ is the artificial gauge field associated with the sinusoidal trajectory of the waveguides, $P, A$ and $\varphi$ are the period, amplitude, and phase of the sinusoidal modulation, respectively. $k_{0}=2 \pi / \lambda$ is the wavenumber in the free space and $k_{x}$ is the spatial momentum in the $x$ direction. $d$ is the center-to-center separation between waveguides and $c$ is the corresponding coupling coefficient between two neighboring straight waveguides (i.e., $A=0)$. Using the high-frequency limit $(2 \pi / P \gg c)$, the 
effective coupling coefficient $c_{\text {eff }}$ can be obtained from Eq. (1)

$$
c_{\text {eff }}(\lambda)=c(\lambda) J_{0}(\eta(\lambda))
$$

where $J_{0}$ is the zero-order Bessel function of the first kind, $\eta(\lambda)=4 \pi^{2} A n(\lambda) d / P \lambda$, and $n(\lambda)$ is the refractive index of the substrate. In Eq. (2), all the parameters are written as the functions of wavelength $\lambda$. The derivative of $c_{\text {eff }}(\lambda)$ to $\lambda$, i.e., the wavelength dispersion writes

$$
\frac{\partial c_{\mathrm{eff}}(\lambda)}{\partial \lambda}=J_{0}(\eta(\lambda)) \frac{\partial c(\lambda)}{\partial \lambda}+J_{1}(\eta(\lambda)) c(\lambda) \frac{\eta(\lambda)}{\lambda}\left[1-\frac{\partial n(\lambda) / \partial \lambda}{n(\lambda) / \lambda}\right]
$$

where $J_{1}$ is the first-order Bessel function of the first kind. According to Eq. (3), the dispersion of effective coupling coefficient $\partial c_{\text {eff }} / \partial \lambda$ consists of two terms, one term is related to the dispersion of straight waveguide (i.e., $J_{0}(\eta(\lambda)) \frac{\partial c(\lambda)}{\partial \lambda}$ ) and the other term represents the dispersion introduced by the artificial gauge field (i.e., $J_{1}(\eta(\lambda)) c(\lambda) \frac{\eta(\lambda)}{\lambda}\left[1-\frac{\partial n(\lambda) / \partial \lambda}{n(\lambda) / \lambda}\right]$ ). Interestingly, the dispersion of straight waveguide can be compensated by the artificial gauge field as long as $J_{0}$ and $J_{1}$ have opposite sign (because $\partial c(\lambda) / \partial \lambda>0$ and $\left.c(\lambda) \frac{\eta(\lambda)}{\lambda}\left[1-\frac{\partial n(\lambda) / \partial \lambda}{n(\lambda) / \lambda}\right]>0\right)$. Figure $1 \mathbf{b}$ shows the Bessel function modulations of $J_{0}$ and $J_{1}$. There indeed exists sign reversal of $J_{0}$ and $J_{1}$ in the red region, which provide the possibility to reduce the dispersion by artificial gauge field. So we strictly calculated the $\partial c_{\text {eff }} / \partial \lambda$ with respect to $\lambda$ and $A$, as shown in Fig. 1c. For $A=0, \partial c_{\text {eff }} / \partial \lambda>0$ and increases with $\lambda$. As $A$ increases, $\partial c_{\text {eff }} / \partial \lambda$ decreases and gets to nearly zero for $A \sim 1 \mu \mathrm{m}$ (the white dashed curve represents $\partial c_{\text {eff }} / \partial \lambda=0$ ), where the dispersion of coupling coefficient can be fully compensated by the artificial gauge field. Figure $1 \mathbf{d}$ shows the effective coupling coefficient as the function of wavelength for different $A$. Indeed, the wavelength dispersion of coupling coefficient gradually becomes flat as $A$ increases (red curve in Fig. 1d), suggesting the insensitivity of the coupling to the wavelength.

To be noted, here the insensitivity to wavelength also indicates the robustness against variations of structural parameters (e.g., waveguide separation $d$ ). It can be seen from the derivative of $c_{\text {eff }}(d)$ to $d$

$$
\frac{\partial c_{\mathrm{eff}}(d)}{\partial d}=J_{0}(\eta(d)) \frac{\partial c(d)}{\partial d}-J_{1}(\eta(d)) c(d) \frac{4 \pi^{2} A n}{P \lambda}
$$


Eq. (4) reveals that the artificial gauge field can introduce compensate term to reduce the structural sensitivity to $d$, as long as $J_{0}$ and $J_{1}$ have opposite sign, which is the same region where the wavelength dispersion can be compensated (red region in Fig. 1b). Figure 1e shows the calculated $\partial c_{\text {eff }} / \partial d$ as functions of $d$ and $A$. It is found that the $\left|\partial c_{\text {eff }} / \partial d\right|$ decreases as $A$ increases and becomes almost zero for $A \sim 1 \mu \mathrm{m}$ (the black dashed curve represents $\partial c_{\text {eff }} / \partial d=0$ ). Figure $1 \mathbf{f}$ shows $c_{\text {eff }}$ as function of $d$ for different $A$ and the flat curve indeed appears at $A=1 \mu \mathrm{m}$ (red curve in Fig. 1f), indicating the insensitivity of coupling to the waveguide separation.
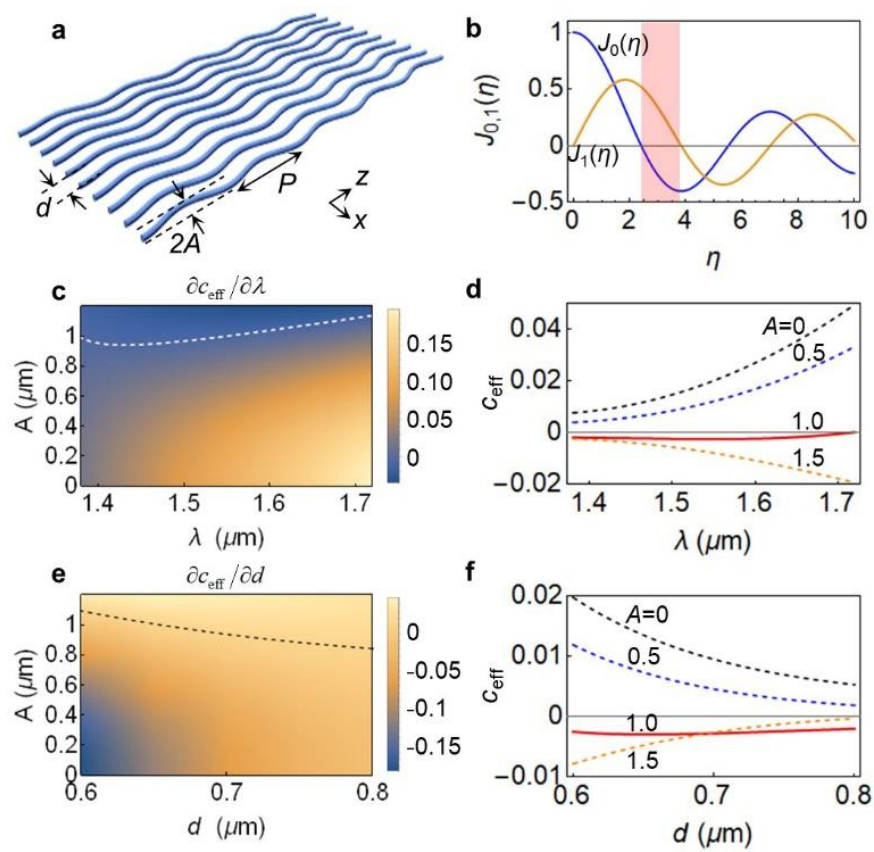

Figure 1| Dispersionless coupling by artificial gauge field. a, Schematic of the 1D waveguides of sinusoidal trajectories with artificial gauge field. b, Zero-order and first-order Bessel modulation of the first kind. There is a sign reversal of $J_{0}(\eta)$ and $J_{1}(\eta)$ in the red region (i.e., dispersion compensation region). c, The derivative of $c_{\text {eff }}$ as a function of $\lambda$ with $d=600 \mathrm{~nm}, P=10 \mu \mathrm{m}$. The white dashed curve represents $\partial c_{\text {eff }} / \partial \lambda=0$. d, Effective coupling coefficient as a function of $\lambda$ for different $A$. From top to bottom, $A=0,0.5,1.0,1.5 \mu \mathrm{m}$. The dispersionless coupling can be obtained for $A=1 \mu \mathrm{m}$. e, The derivative of $c_{\text {eff }}$ as a function of $d$ with $\lambda=1550 \mathrm{~nm}, P=10 \mu \mathrm{m}$. The black dashed curve represents $\partial c_{\text {eff }} / \partial d=0$. f, Effective coupling coefficient as a function of $d$ for different $A$. From top to bottom, $A=0,0.5,1.0,1.5 \mu \mathrm{m}$. The insensitivity of coupling to the waveguide separation can be obtained for $A=1 \mu \mathrm{m}$.

\section{Gauge field controlled coupling in densely packed waveguides}

To be mentioned, our strict analyses are based on weakly coupling conditions, which is inappropriate for strongly coupled dense silicon waveguides. Nevertheless, it still provides the foundation to investigate the 
effect of artificial gauge field in strong coupling regimes. On a Si-on-insulator (SOI) platform, the designed silicon waveguide is $400 \mathrm{~nm}$ wide and $220 \mathrm{~nm}$ thick, allowing a fundamental waveguide mode at $\lambda=1550 \mathrm{~nm}$. The waveguide separation $d=600 \mathrm{~nm}$ is smaller than $\lambda / 2$. A commercial finite-element analysis solver (Comsol Multiphysics 5.3) was employed for full-wave simulations. Figure 2a shows the simulated and theoretical (Eq. (2)) coupling coefficient as a function of $\eta$, which both decrease to zero then increase inversely as $\eta$ increases. Differently, high-density of silicon waveguides can lead to two major consequences compared to the theoretical results: (1) much deeper coupling modulations, i.e., the strength of negative coupling can be larger than the positive coupling in straight waveguides, suggesting the reduced coupling length; (2) much wider dispersion compensation region (red arrows for strong coupling regime, blue for weakly coupling), indicating wider dispersion flat band. Specifically, Figure $2 \mathbf{c}$ shows the simulated coupling coefficient as a function of wavelength for different $A$. The coupling strength drastically changes as large as $0.070 \mu \mathrm{m}^{-1}$ over $1400-1600 \mathrm{~nm}$ waveband for the conventional straight waveguides without artificial gauge field $(A=0)$. However, when the artificial gauge field is introduced by nonzero $A$, the coupling coefficient between the two waveguides gradually becomes negative, and the change of coupling with respect to wavelength gradually slows down. In particular, when $A=A_{\mathrm{c}}=0.9 \mu \mathrm{m}$, the wavelength dispersion curve almost becomes flat with largest coupling deviations smaller than $0.010 \mu \mathrm{m}^{-1}$ over the same $1400-1600 \mathrm{~nm}$ waveband, $\sim 1 / 7$ of the conventional case without artificial gauge field. Importantly, the value of $A_{\mathrm{c}}$ where the flat band appears can be tuned by changing the waveguide separation $d$ (see Fig. 2b), and different dispersionless coupling strengths $c_{\mathrm{c}}$ can be obtained (see Supplementary Fig. S1 for details). Besides, we also simulated the effective coupling coefficient as a function of $d$ for $\lambda=1550 \mathrm{~nm}$ to illustrate the insensitivity to waveguide structural parameters, as shown in Fig. 2d. It is found that when $A \sim 0.9 \mu \mathrm{m}$, the coupling coefficient will remain stable with the largest deviation less than $0.0054 \mu \mathrm{m}^{-1}$ despite a large variation of $d(600-750 \mathrm{~nm})$. As a comparison, for traditional straight waveguide coupling situation without artificial gauge field $(A=0)$, the coupling undergoes drastically changes $\sim 0.056 \mu \mathrm{m}^{-1}$ for the same variation of $d$, nearly ten times of the case with artificial gauge field. We further show the simulated propagations of the optical field in the silicon waveguides without $(A=0)$ and with the AGF $(A=0.9 \mu \mathrm{m})$ (see Supplementary Fig. S2 and Fig. S3). It is shown that the optical field can always couple to another waveguide at a distance $(\sim 34 \mu \mathrm{m})$ for $A=0.9 \mu \mathrm{m}$ over a wide wavelength band (1400-1700 nm) and with a large structural tolerance (550-800 nm). However, 
the performance of the traditional straight waveguides without artificial gauge field changes drastically.
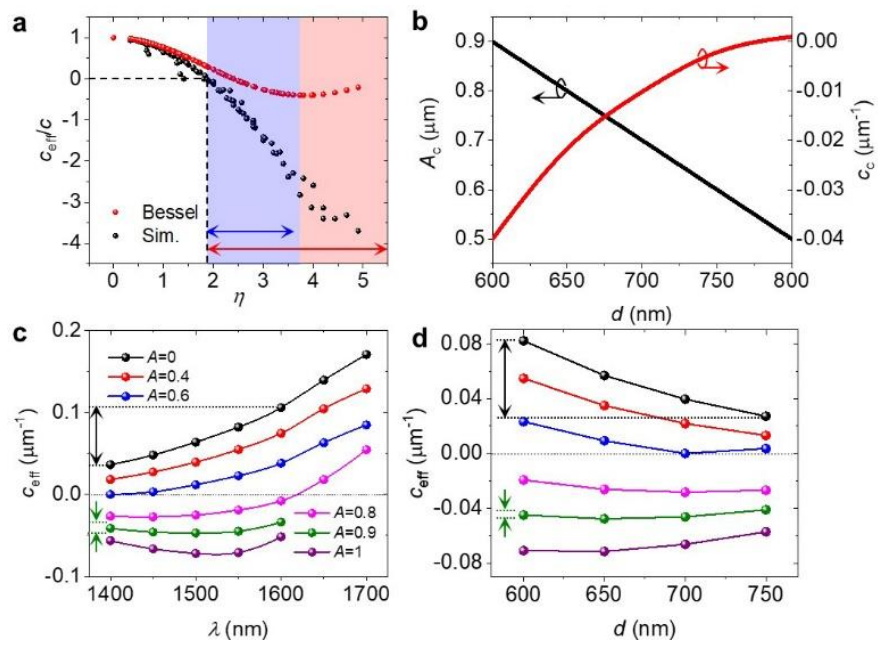

\begin{abstract}
Figure 2 | Introducing artificial gauge field into high-density silicon platform. a, Simulated (black) and Bessel modulated (red) effective coupling coefficient as a function of $\eta$. The strength of negative coupling (black dots) can be larger than the positive coupling in straight waveguides. Besides, the sign reversal region expands (red arrow) as compared to the Bessel modulations (blue arrow). $\mathbf{b}, A_{\mathrm{c}}$ and $c_{\mathrm{c}}$ as the function of $d$. The dispersionless coupling strength $c_{\mathrm{c}}$ can be tuned by choosing different $d$. c, Simulated effective coupling coefficient as a function of $\lambda$ for $d=600 \mathrm{~nm}$. d, Simulated effective coupling coefficient as a function of $d$ for $\lambda=1550 \mathrm{~nm}$. The period of gauge modulation is fixed to $P=10 \mu \mathrm{m}$.
\end{abstract}

\title{
Experimental confirmations
}

To confirm our theoretical finding, we fabricated the AGF-based waveguide devices $(A=0.9 \mu \mathrm{m})$ with $d=600 \mathrm{~nm}$ and $34.5 \mu \mathrm{m}$ length (see Figs. 3a,b) to achieve directional coupler (DC) functions by electron beam lithography and inductively coupled plasma (ICP) etching (see Methods). A near-infrared laser at the wavelength range of $1400-1650 \mathrm{~nm}$ was input to the Port- $\mathrm{I}_{1}$ through a single-mode fiber, then the transmission signal of Port- $\mathrm{O}_{1}$ and Port- $\mathrm{O}_{2}$ were collected by another multi-mode fiber, which was connected to a spectrometer (YOKOGAWA AQ6375) to measure the output intensity $\left(I_{\mathrm{O} 1}\right.$ and $\left.I_{\mathrm{O} 2}\right)$. Another near-infrared camera (Xeva-1.7-320) was used to image the coupling-in and -out processes through a microscope objective (see Methods). A conventional strip waveguide device without artificial gauge field was also fabricated for comparison, which was designed to work at $1550 \mathrm{~nm}$ (Fig. 3c). The experimentally measured coupling ratio $\left(I_{\mathrm{O} 2} /\left(I_{\mathrm{O} 2}+I_{\mathrm{O} 1}\right)\right)$ for the devices with (black) and without (red) artificial gauge field is shown in Fig. 3e, and the 
corresponding output images for different wavelengths are shown in Fig. 3d. The light input from Port- $\mathrm{I}_{1}$ all couples to the Port- $\mathrm{O}_{2}$ for AGF devices over 1450-1650 nm band with the largest deviation less than $2 \mathrm{~dB}$. Yet it deviates $\sim 20 \mathrm{~dB}$ for the same wavelength band for conventional devices. We do not observe a significant difference in transmitted optical power between the AGF and conventional devices. For example, we obtained low insertion loss data of $0.5 \mathrm{~dB}$ for the AGF coupler and $0.3 \mathrm{~dB}$ for the conventional one (averaged over three samples) by comparing the transmitted power to that of a referenced single straight waveguide. Besides, we designed and fabricated a 3-dB coupler with 50\%:50\% splitting ratio, as the experimental results shown in Figs. 3f,g. The splitting performance is stable with respect to wavelengths for the AGF coupler, and the largest deviation is also less than $2 \mathrm{~dB}$. As a comparison, the traditional straight device only works at the designed $1550 \mathrm{~nm}$ and exhibits large wavelength dependence. Furthermore, we also fabricated a set of AGF and straight devices with different waveguide separation $d$ to verify the robustness against structural variations. It is shown that the deviation of $\sim 150 \mathrm{~nm}$ of $d$ can hardly affect the coupling and splitting performance of the AGF coupler, while it loses the functions completely even for several ten nanometers deviations for the straight ones (see Supplementary Sec. I and Fig. S4). This robustness promises large tolerance to dimensional uncertainties due to the fabrication, thus allowing scaling to large circuits.
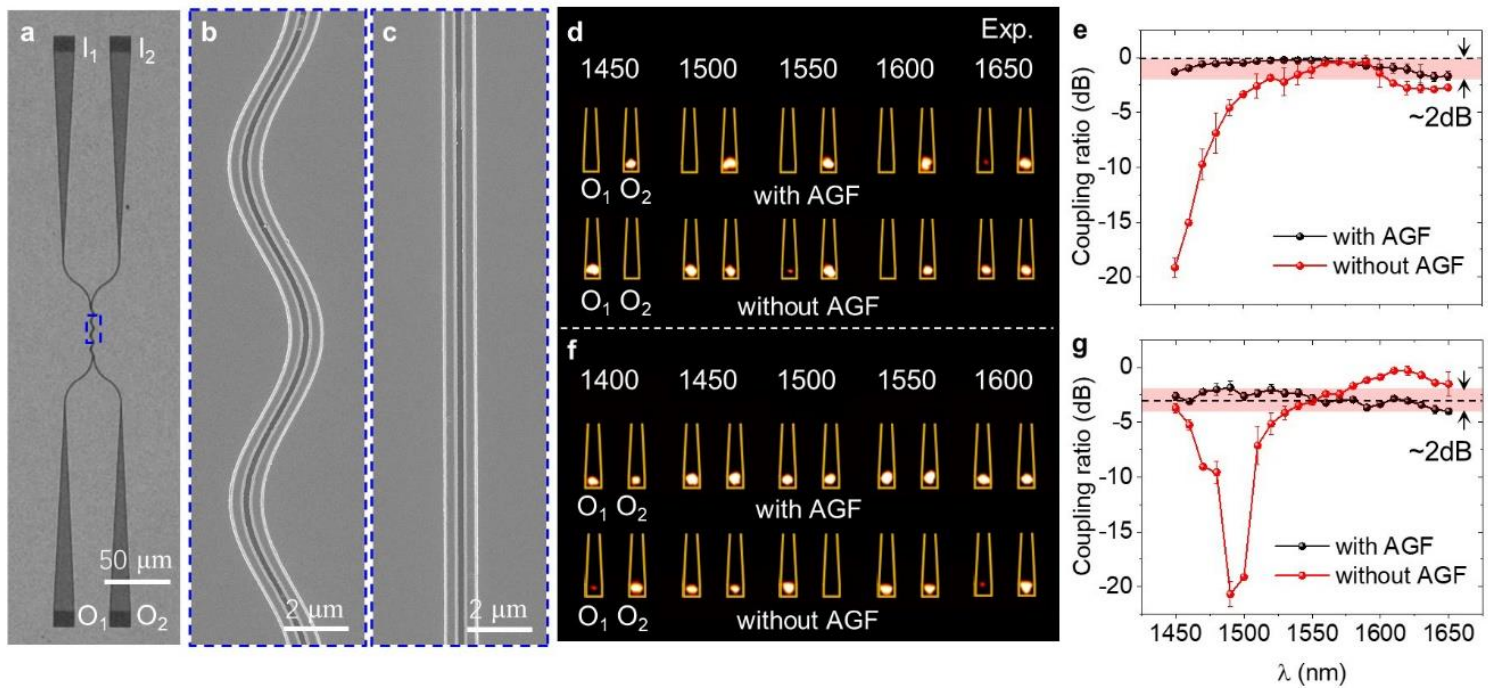

Figure 3 | Experimental demonstrations of dispersionless coupling with artificial gauge field on silicon platform. a, A microscope image of the fabricated samples on silicon platform. b,c, Zoomed-in scanning electron microscopy image of AGF (b) and conventional (c) coupler devices. d, Experimentally detected output scattering fields from Port- $\mathrm{O}_{1}$ and Port- $\mathrm{O}_{2}$ in AGF and conventional directional coupler. The bright spots from the expected output Port- $\mathrm{O}_{2}$ are evidently observed for different wavelength for the AGF coupler. e, Coupling ratio of the Port- 
$\mathrm{O}_{2}$ as a function of wavelength. f,g, Corresponding results for the $3 \mathrm{~dB}$ couplers, which has size of $17.5 \mu \mathrm{m}$. The two output ports have almost equal intensities for different wavelengths for the samples with AGF.

\section{Three-level cascaded waveguides network}

To further show the potential of AGF-assisted devices in massive integration, we demonstrate the optical routing functionalities of AGF-based devices in three-level cascaded waveguides networks, as the schematics shown in Fig. 4a, which consists of seven unit devices with two input ports $\left(I_{1}\right.$ and $\left.I_{2}\right)$ and eight output ports $\left(\mathrm{O}_{1}, \mathrm{O}_{2}, \ldots, \mathrm{O}_{8}\right)$. The units can be the directional coupler or the $3 \mathrm{~dB}$ coupler. When the light was input from Port- $\mathrm{I}_{1}$, it was expected to be routed to the edge port $\left(\mathrm{O}_{8}\right)$ for the directional coupler cascaded systems, while it should be equally distributed to all ports for the $3 \mathrm{~dB}$ coupler networks. Figure $4 \mathbf{b}$ shows the experimentally detected output scattering fields for the directional coupler cascaded systems, in which the light is routed to the preferred port $\left(\mathrm{O}_{8}\right)$ for the AGF devices with respect to different wavelengths. However, for the conventional system without AGF, it cannot work well even at the designed wavelength $1550 \mathrm{~nm}$ and the lights come from different output ports as the wavelength changes. As for the $3 \mathrm{~dB}$ coupler cascaded networks, the eight output ports share almost equal intensities for the AGF system with different wavelengths, while for the straight case, the intensities of eight output ports are chaotic as the wavelength changes (see Fig. 4d). Figures $4 \mathbf{c}, \mathbf{e}$ show the experimental data of coupling ratio for the AGF and straight devices with respect to different wavelength $\lambda$. It is observed that coupling ratio of AGF coupler $\left(I_{\mathrm{O} 8} /\left(I_{\mathrm{O} 1}+\ldots+I_{\mathrm{O} 8}\right)\right)$ keeps a high value in a considerably wide bandwidth $(1400 \sim 1600 \mathrm{~nm})$, with the largest deviation about $6 \mathrm{~dB}$ at $1400 \mathrm{~nm}$. Yet there is a large discrepancy for the conventional device (>24.7dB at $1400 \sim 1450 \mathrm{~nm})$, and it performs not well even at the designed wavelength $1550 \mathrm{~nm}(\sim-3.58 \mathrm{~dB})$. As for the $3 \mathrm{~dB}$ case, the coupling ratio of each port $\left(I_{\mathrm{O} i}\left(\left(I_{\mathrm{O} 1}+\ldots+I_{\mathrm{O} 8}\right)\right)\right.$ remains $\sim-9 \mathrm{~dB}$ over nearly $200 \mathrm{~nm}$ wavelength band for the AGF device, and the largest deviation $\sim 6 \mathrm{~dB}$. However, for the straight case, it even exhibits $5 \mathrm{~dB}$ deviation for the designed 1550 $\mathrm{nm}$ wavelength and reaches the maximum deviations $\sim 21 \mathrm{~dB}$ as the wavelength varies. Our results show the potential of the AGF-assisted waveguides in large-scale and dense photonic integration. 

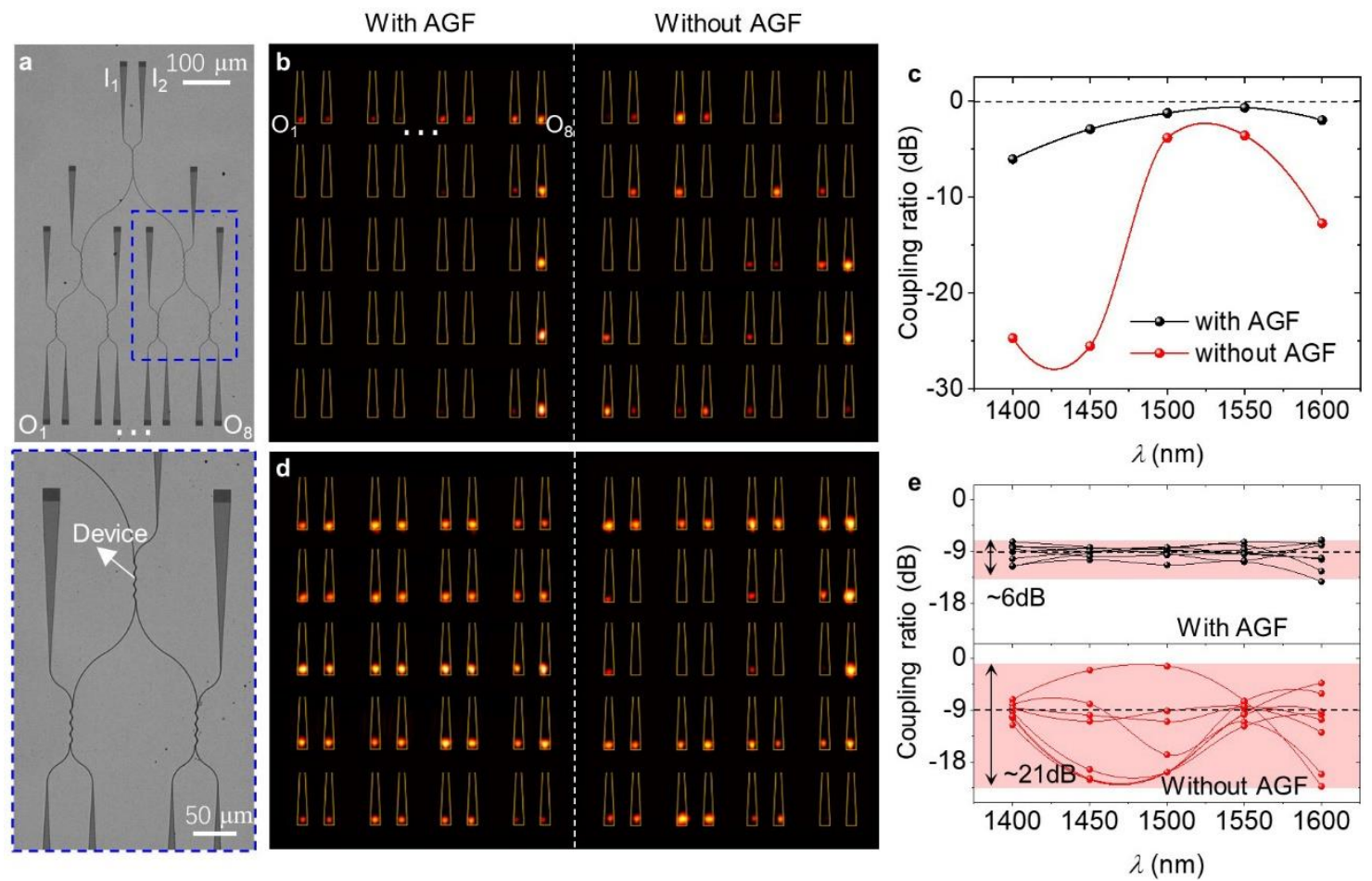

Figure 4 | Three-level cascaded AGF device network. a, Microscope images of the fabricated three-level cascaded waveguides networks. b, Experimentally detected output scattering fields in AGF and conventional directional coupler cascaded systems for different wavelength. From top to bottom, $\lambda=1400,1450,1500,1550$, $1600 \mathrm{~nm}$. It is observed that the light can be routed to the expected Port- $\mathrm{O}_{8}$ for different wavelength in the AGF networks. c, Coupling ratio for the Port- $\mathrm{O}_{8}$ as a function of wavelength for directional coupler cascaded samples. d,e, Corresponding results for $3 \mathrm{~dB}$ coupler cascaded samples. The eight output ports share almost the same intensities for the AGF system as the wavelength changes.

\section{Discussion}

To be noted, there have been similar approaches using bending waveguides for broadband coupling ${ }^{39-41}$. However, they were only demonstrated in particular cases without providing a general theory. Here we systemically reveal the physical origin of the dispersionless coupling among curved waveguides, which can be well interpreted by the artificial gauge field theory. In this regard, we definitely provide a convincing theoretical framework that well includes previous bending designs. More importantly, the demonstrated coupler devices in this work designed by the AGF theory exhibit much better performances (including broader working bandwidth and more robustness against structural variations).

Furthermore, our work provides a general design strategy that will not limit to the coupler devices, but 
also work for other functionalities, e.g., the broadband elimination of crosstalk among densely arranged waveguides. It has been in a long pursuit of a dense waveguide integration for light propagation without $\operatorname{crosstalk}^{20,42,43}$. According to the crossover of coupling coefficient from positive to negative in conventional curved waveguides due to the Bessel function property ${ }^{42,43}$, there is only one zero-coupling point for one particular wavelength. Our approach breaks this limit and produces a flat zero-coupling band, and thus gives rise to a broadband zero-coupling for densely arranged waveguides (e.g., $d=770 \mathrm{~nm}, A=0.51 \mu \mathrm{m}$, see Fig. 2b). We provide the detailed results in Supplementary Sec. II and Fig. S5, in which a broadband (bandwidth $>100 \mathrm{~nm}$ ) low-crosstalk (extinction ratio $>10 \mathrm{~dB}$ ) wave-guiding are demonstrated both in theory and experiments. The sub-half-wavelength pitch size $(d=770 \mathrm{~nm})$ indicates its high potential applications in densely photonic integrations in a broadband scheme, e.g., compact photonic routings, optical-phased array, etc.

\section{Conclusion}

In conclusion, we have demonstrated the coupling dispersion engineering among waveguides by artificial gauge field and proposed an AGF-based broadband optical waveguides coupler, which greatly reduces sensitivity and dependence on the wavelength and structural variations under high-density integration. According to our data, the AGF devices show good coupling functionality for structural deviation as large as $150 \mathrm{~nm}(75 \%)$ and a wavelength range $\sim 200 \mathrm{~nm}$ centered at the $1550 \mathrm{~nm}$, while the device size $(20-30 \mu \mathrm{m})$ and insertion loss $(0.4-0.6 \mathrm{~dB})$ can be kept comparable small as the straight waveguides. We experimentally demonstrated the advantages by comparison with conventional devices in large-scale three-leveled cascaded device networks. This work introduces the artificial gauge field for dispersion control of integrated devices, which would open a new avenue for robust and broadband large-scale integrations with high-density.

\section{Methods}

Sample preparation. The waveguide devices are fabricated on a standard $220 \mathrm{~nm}$ silicon-on-insulator (SOI) wafer with a $2 \mu \mathrm{m}$ buried dioxide buffer layer using electron beam lithography and inductively coupled plasma (ICP) etching. Then $220 \mathrm{~nm}$ AR-N7520 photoresist film is spin-coated onto the substrate and baked at $85^{\circ} \mathrm{C}$ for $1 \mathrm{~min}$. After that, the sample is exposed to electron beam in E-beam writer (Elionix, ELS-F125) 
and developed to form the AR-N7520 nanostructures. Then, the sample transferred into HSE Series Plasma Etcher 200 and etched with C4F8 and SF6 (the flow rates of these two types of gases are 75sccm:30sccm). After the ICP etching, the remaining AR-N7520 is removed by using an $\mathrm{O}_{2}$ plasma for 5 minutes.

Optical measurements. In optical measurements, a tunable laser source (N7778C) with a wavelength range from $1450 \mathrm{~nm}$ to $1650 \mathrm{~nm}$ was used. The light with different wavelengths was focused at the input grating by a single-mode fiber, and then coupled into the waveguide mode. The output signals are collected by a multi-mode fiber and connected to a spectrometer (YOKOGAWA AQ6375). The light spot image was detected by the scattering field from the output end by a near-infrared camera (Xeva-1.7-320) through a microscope objective $(50 \times)$.

\section{Funding}

This work was supported by the National Key Research and Development Program of China (2017YFA0303701, 2016YFA0202103); National Natural Science Foundation of China (91850204). Tao Li thanks the support from Dengfeng Project B of Nanjing University.

\section{Acknowledgments}

We thank the micro-fabrication center of the National Laboratory of Solid State Microstructures (NLSSM) for technique support.

\section{Author contributions}

T.L. and W.S. conceived the idea. W.S. proposed the theoretical design, performed the numerical design; S.G. fabricated the sample; W.S. and Ting L. performed the optical measurement with the help of Z.W., S.W. and C.C.; T.L. organized the project. W.S. and T.L. analyzed the results and wrote the paper. All authors discussed the results and commented on the manuscript.

Competing financial interests: The authors declare no conflicts of interest. 


\section{References}

1. Miller, S. E. Integrated Optics: An Introduction. Bell System Tech. J. 48, 2059 (1969).

2. Nagarajan, R. Large-scale photonic integrated circuits. IEEE J. Sel. Top. Quantum Electron. 11, 50-65 (2005).

3. Soref, R. The past, present, and future of silicon photonics. IEEE J. Sel. Top. Quantum Electron. 12, 1678-1687 (2006).

4. Dai, D., Bauters, J. \& Bowers, J. E. Passive technologies for future large-scale photonic integrated circuits on silicon: polarization handling, light non-reciprocity and loss reduction. Light Sci. Appl. 1, e1 (2012).

5. Shen, Y. et al. Deep learning with coherent nanophotonic circuits. Nat. Photon. 11, 441-446 (2017).

6. Kim, S. et al. Dispersion engineering and frequency comb generation in thin silicon nitride concentric microresonators. Nat. Commun. 8, 372 (2017).

7. Born, M. \& Wolf, E. Principles of Optics. 7th ed. (Cambridge Press, 1999).

8. Bennett, G. A. \& Chen, C.-L. Wavelength dispersion of optical waveguides. Appl. Opt. 19, 1990-1995 (1980).

9. Xu, Q., Schmidt, B., Pradhan, S. \& Lipson, M. Micrometre-scale silicon electro-optic modulator. Nature 435, 325-327 (2005).

10. Reed G. T., Mashanovich, G., Gardes, F. Y. \& Thomson, D. J. Silicon optical modulators. Nat. Photon 4, 518-526 (2010).

11. Yariv, A. Coupled-mode theory for guided-wave optics. IEEE J. Quantum Electron. 9, 919-933 (1973).

12. Mrejen, M. et al. Adiabatic elimination-based coupling control in densely packed subwavelength waveguides. Nat. Commun. 6, 7565 (2015).

13. Oukraou, H., Coda, V., Rangelov, A. A., \& Montemezzani, G. Broadband photonic transport between waveguides by adiabatic elimination. Phys. Rev. A 97, 023811 (2018).

14. Yun, H., Shi, W., Wang, Y., Chrostowski, L. \& Jaeger, N. A. F. $2 \times 2$ adiabatic 3-db coupler on siliconon-insulator rib waveguides. Proceedings of SPIE-The International Society for Optical Engineering (2013).

15. Molesky, S. et al. Inverse design in nanophotonics. Nat. Photon. 12, 659-670 (2018). 
16. Piggott, A. Y. et al. Inverse design and demonstration of a compact and broadband on-chip wavelength demultiplexer. Nat. Photon. 9, 374-377 (2015).

17. Shen, B., Wang, P., Polson, R. \& Menon, R. An integrated-nanophotonics polarization beamsplitter with $2.42 .4 \mathrm{~mm}^{2}$ footprint. Nat. Photon. 9, 378-382 (2015).

18. Shen, B., Polson, R. \& Menon, R. Increasing the density of passive photonic-integrated circuits via nanophotonic cloaking. Nat. Commun. 7, 13126 (2016).

19. Li, Z. et al. Controlling propagation and coupling of waveguide modes using phase-gradient metasurfaces. Nat. Nanotech. 12, 675-683 (2017).

20. Jahani, S. et al. Controlling evanescent waves using silicon photonic all-dielectric metamaterials for dense integration. Nat. Commun. 9, 1893 (2018).

21. Cheben, P., Halir, R., Schmid, J. H., Atwater, H. A. \& Smith, D. R. Subwavelength integrated photonics. Nature 560, 565-572 (2018).

22. Halir, R. et al. Colorless directional coupler with dispersion engineered sub-wavelength structure, Opt. Exp. 20, 13470-13477 (2012).

23. Mia, M. B. et al. Exceptional coupling in photonic anisotropic metamaterials for extremely low waveguide crosstalk. Optica 7, 881-887 (2020).

24. Song, W. et al. Robust and Broadband Optical Coupling by Topological Waveguide Arrays. Laser Photon. Rev. 14, 1900193 (2020).

25. Wang, Y. et al. Coherent Interactions in One-Dimensional Topological Photonic Systems and Their Applications in All-Optical Logic Operation. Nano Lett. 20, 8796-8802 (2020).

26. Fang, K., Yu, Z. \& Fan, S. Realizing effective magnetic field for photons by controlling the phase of dynamic modulation. Nat. Photon. 6, 782-787 (2012).

27. Fang, K., Yu, Z. \& Fan, S. Photonic Aharonov-Bohm effect based on dynamic modulation. Phys. Rev. Lett. 108, 153901 (2012).

28. Longhi, S. et al. Observation of dynamic localization in periodically curved waveguide arrays. Phys. Rev. Lett. 96, 243901 (2006).

29. Szameit, A. et al. Polychromatic dynamic localization in curved photonic lattices. Nat. Phys. 5, 271-275 (2009). 
30. Szameit, A. et al. Observation of two-dimensional dynamic localization of light. Phys. Rev. Lett. 104, 223903 (2010).

31. Rechtsman, M. C. et al. Photonic Floquet topological insulators. Nature 496, 196-200 (2013).

32. Lustig, E. et al. Photonic topological insulator in synthetic dimensions. Nature 567, 356-360 (2019).

33. Song, W. et al. Gauge-induced Floquet topological states in photonic waveguides. arXiv: 2010.05408.

34. Zeuner, J. M. et al. Optical analogues for massless Dirac particles and conical diffraction in one dimension. Phys. Rev. Lett. 109, 023602 (2012).

35. Song, W. et al. Demonstration of imaginary-mass particles by optical simulation in non-Hermitian systems. arXiv: 2011.08496.

36. Lumer, Y. et al. Light guiding by artificial gauge fields. Nat. Photon. 13, 339-345 (2019).

37. Lin, Q. \& Fan, S. Light guiding by effective gauge field for photons. Phys. Rev. X 4, 031031 (2014).

38. Song, W. et al. Sub-wavelength self-imaging in cascaded waveguide arrays. Adv. Photon. 2, 036001 (2020).

39. Morino, H., Maruyama, T., \& Iiyama, K. Reduction of wavelength dependence of coupling characteristics using Si optical waveguide curved directional coupler. J. Lightwave Tech. 32, 2188-2192 (2014).

40. Chen, S., Shi, Y., He, S., \& Dai, D. Low-loss and broadband $2 \times 2$ silicon thermo-optic Mach-Zehnder switch with bent directional couplers. Opt. Lett. 41, 836-839 (2016).

41. Chen, G. F. et al. Broadband silicon-on-insulator directional couplers using a combination of straight and curved waveguide sections. Sci. Rep. 7, 1-8 (2017).

42. Zhang, F. et al. Sinusoidal anti-coupling SOI strip waveguides. In CLEO: Science and Innovations SM1I-7 (Optical Society of America, 2015).

43. Yi, X., Zeng, H., Gao, S., \& Qiu, C. Design of an ultra-compact low-crosstalk sinusoidal silicon waveguide array for optical phased array. Opt. Express 28, 37505-37513 (2020). 

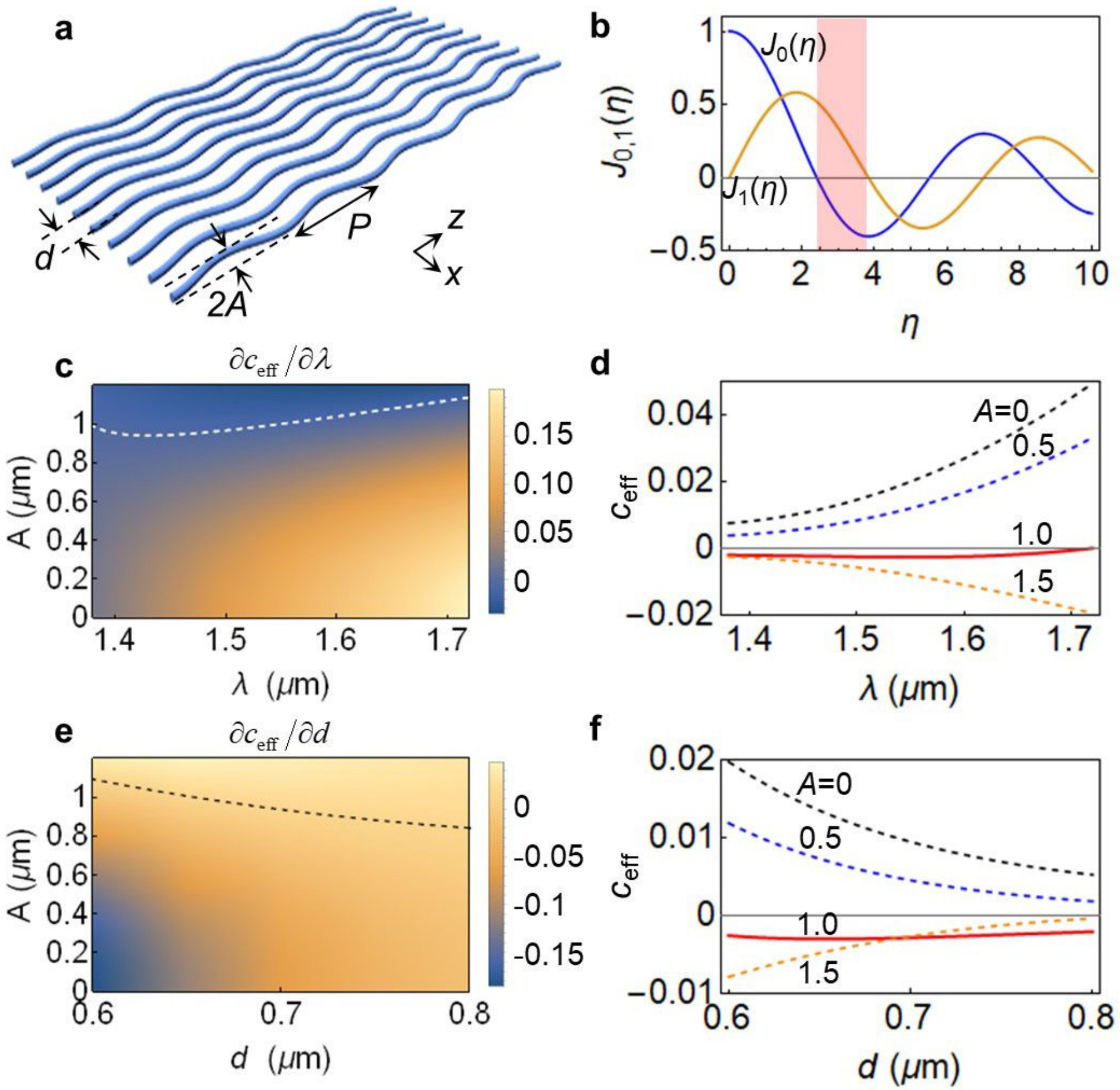

Figure 1

Dispersionless coupling by artificial gauge field. a, Schematic of the 1D waveguides of sinusoidal trajectories with artificial gauge field. b, Zero-order and first-order Bessel modulation of the first kind. There is a sign reversal of $\mathrm{JO}(\eta)$ and $\mathrm{J} 1(\eta)$ in the red region (i.e., dispersion compensation region). c, The derivative of ceff as a function of $\lambda$ with $d=600 \mathrm{~nm}, P=10 \mu \mathrm{m}$. The white dashed curve represents $\partial$ ceff/ $\partial \lambda=0$. $d$, Effective coupling coefficient as a function of $\lambda$ for different $A$. From top to bottom, $A=0,0.5,1.0$, 
$1.5 \mu \mathrm{m}$. The dispersionless coupling can be obtained for $A=1 \mu \mathrm{m}$. e, The derivative of ceff as a function of $d$ with $\lambda=1550 \mathrm{~nm}, P=10 \mu \mathrm{m}$. The black dashed curve represents $\partial$ ceff $/ \partial \mathrm{d}=0$. $\mathrm{f}$, Effective coupling coefficient as a function of $d$ for different $A$. From top to bottom, $A=0,0.5,1.0,1.5 \mu \mathrm{m}$. The insensitivity of coupling to the waveguide separation can be obtained for $A=1 \mu \mathrm{m}$.

a
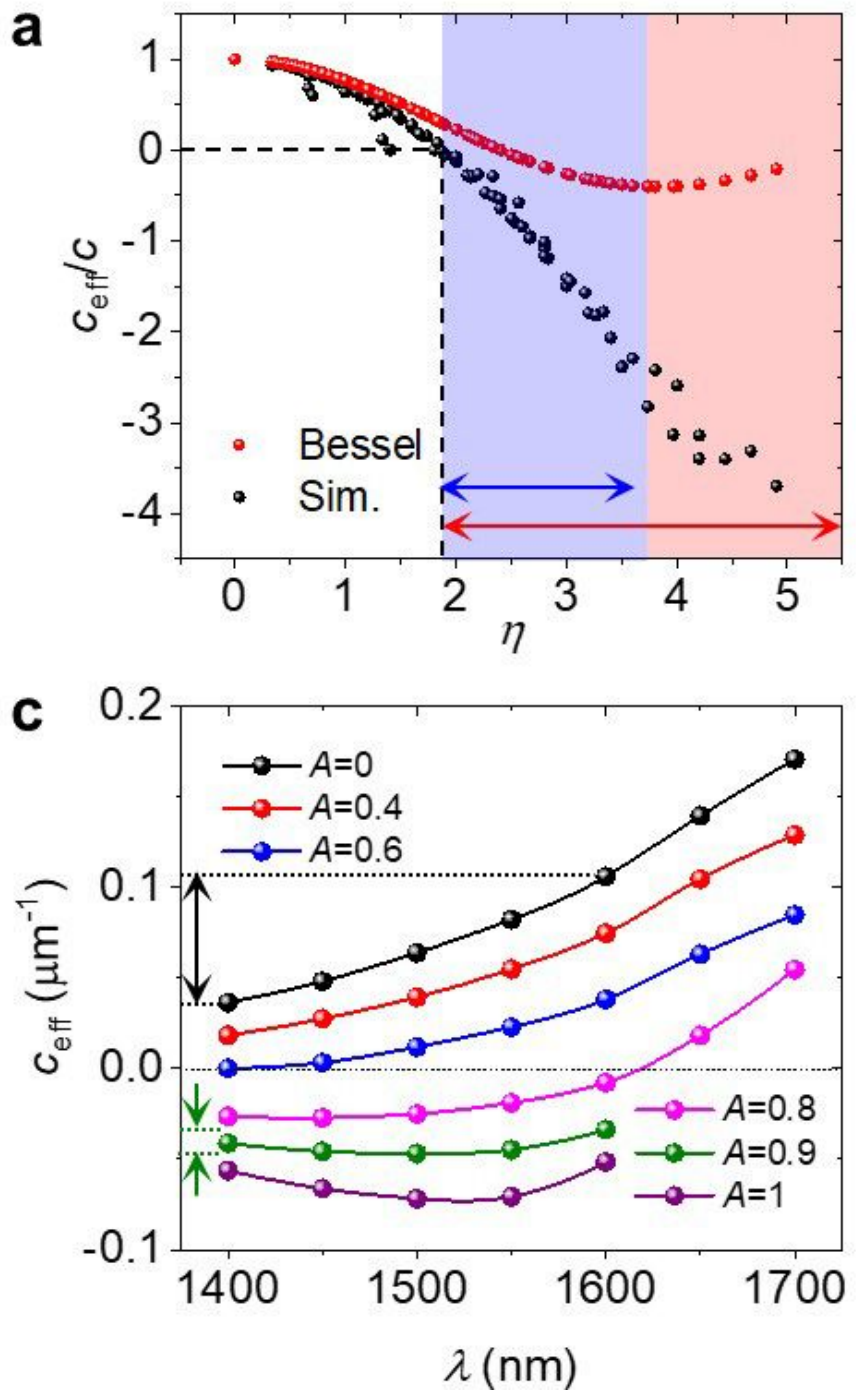

b
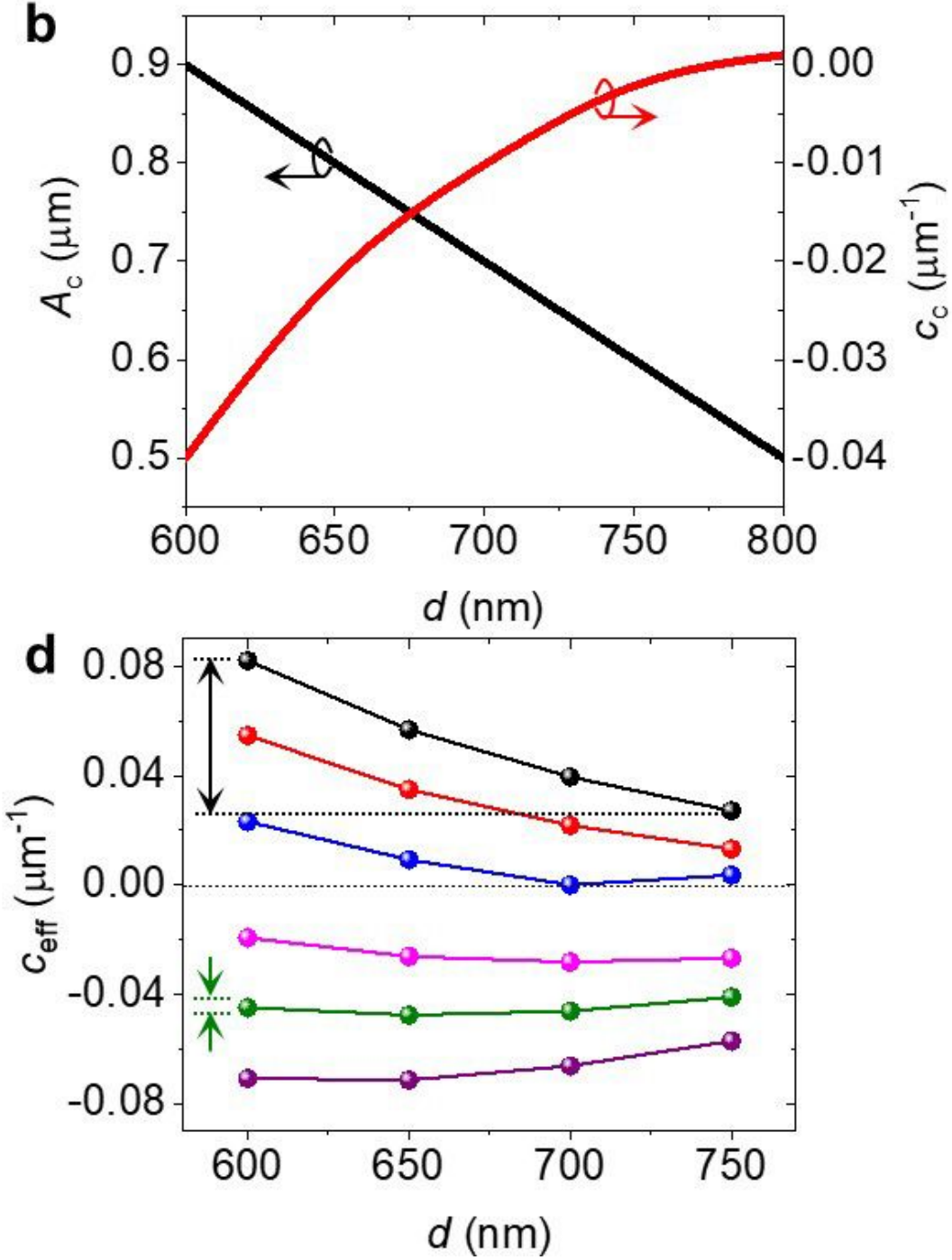

Figure 2

Introducing artificial gauge field into high-density silicon platform. a, Simulated (black) and Bessel modulated (red) effective coupling coefficient as a function of $\eta$. The strength of negative coupling (black dots) can be larger than the positive coupling in straight waveguides. Besides, the sign reversal region expands (red arrow) as compared to the Bessel modulations (blue arrow). b, Ac and cc as the function of $d$. The dispersionless coupling strength cc can be tuned by choosing different d. c, Simulated effective coupling coefficient as a function of $\lambda$ for $d=600 \mathrm{~nm}$. $d$, Simulated effective coupling coefficient as a function of $d$ for $\lambda=1550 \mathrm{~nm}$. The period of gauge modulation is fixed to $P=10 \mu \mathrm{m}$. 

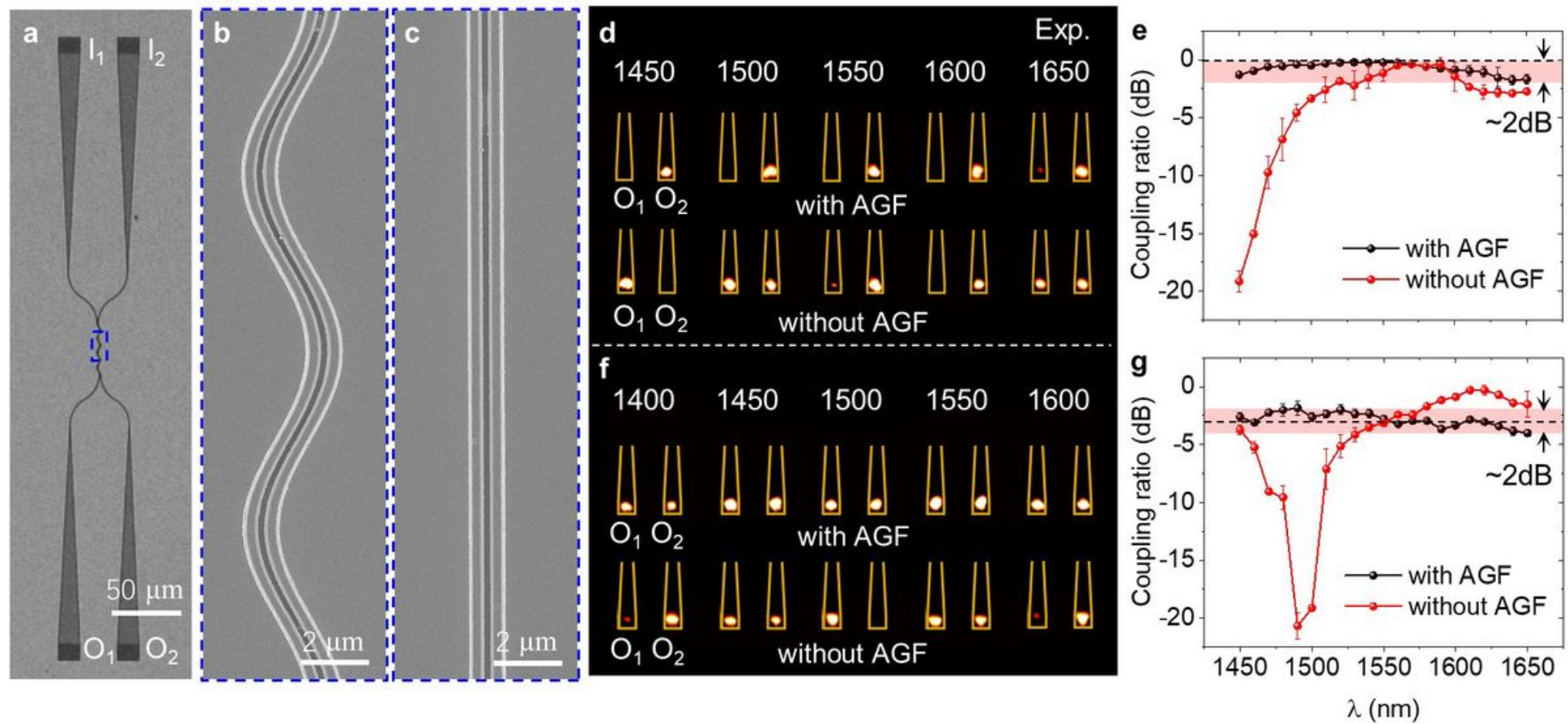

\section{Figure 3}

Experimental demonstrations of dispersionless coupling with artificial gauge field on silicon platform. a, A microscope image of the fabricated samples on silicon platform. b,c, Zoomed-in scanning electron microscopy image of AGF (b) and conventional (c) coupler devices. d, Experimentally detected output scattering fields from Port-01 and Port-02 in AGF and conventional directional coupler. The bright spots from the expected output Port-02 are evidently observed for different wavelength for the AGF coupler. e, Coupling ratio of the Port- 02 as a function of wavelength. $\mathrm{f}, \mathrm{g}$, Corresponding results for the $3 \mathrm{~dB}$ couplers, which has size of $17.5 \mu \mathrm{m}$. The two output ports have almost equal intensities for different wavelengths for the samples with AGF. 

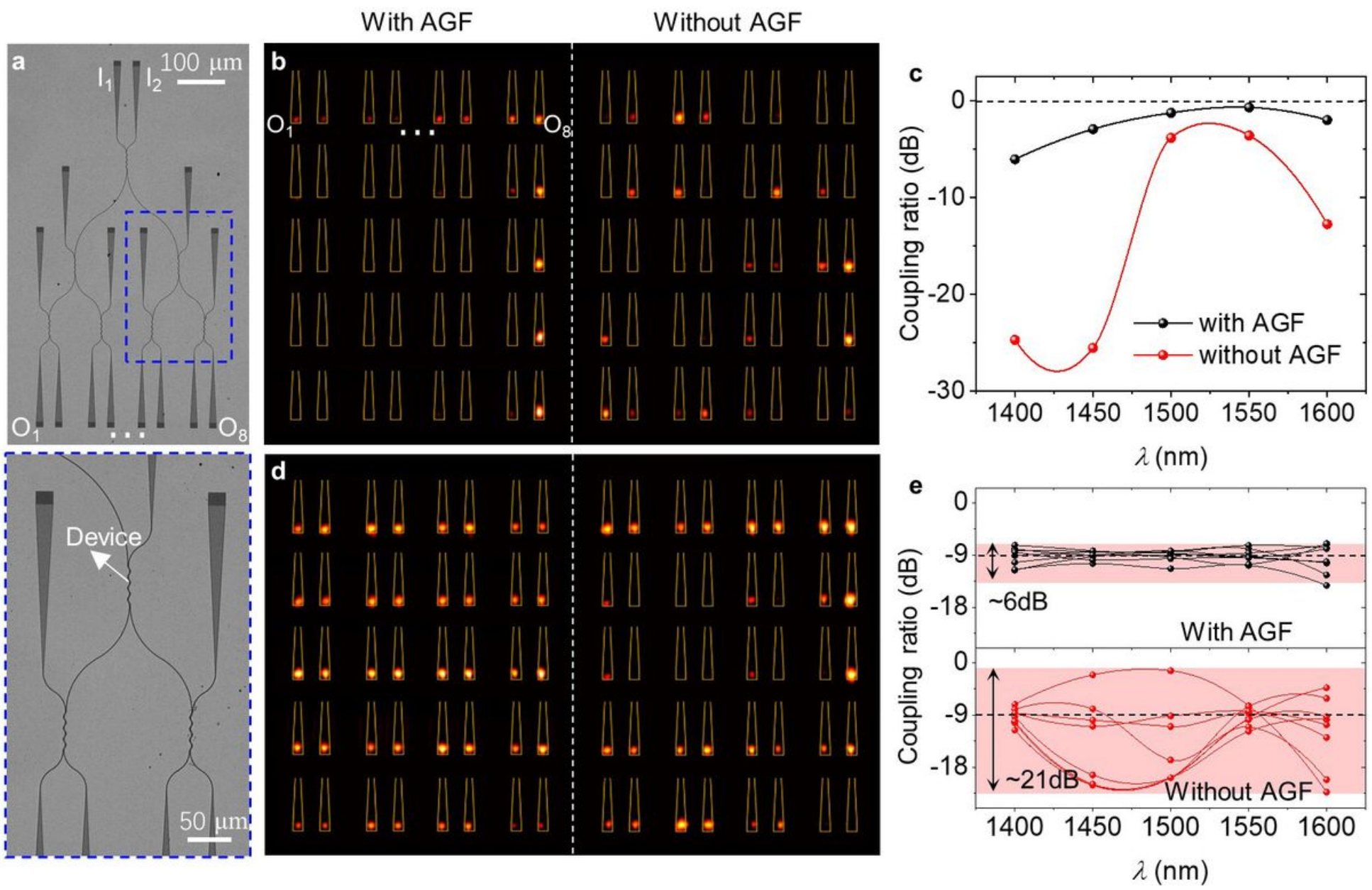

\section{Figure 4}

Three-level cascaded AGF device network. a, Microscope images of the fabricated three-level cascaded waveguides networks. b, Experimentally detected output scattering fields in AGF and conventional directional coupler cascaded systems for different wavelength. From top to bottom, $\lambda=1400,1450,1500$, $1550,1600 \mathrm{~nm}$. It is observed that the light can be routed to the expected Port-08 for different wavelength in the AGF networks. C, Coupling ratio for the Port-08 as a function of wavelength for directional coupler cascaded samples. $d, e$, Corresponding results for $3 \mathrm{~dB}$ coupler cascaded samples. The eight output ports share almost the same intensities for the AGF system as the wavelength changes.

\section{Supplementary Files}

This is a list of supplementary files associated with this preprint. Click to download.

- supplementalsongetal.docx 